\title{
Evidence for improvements to risk stratification in high-risk gastrointestinal stromal tumor patients
}

This article was published in the following Dove Press journal: Gastrointestinal Cancer:Targets and Therapy

\author{
Alessandra Greco' \\ Sabrina Rossi ${ }^{2}$ \\ Cesare Ruffolo' \\ Bruno Pauletti ${ }^{3}$ \\ Angelo Paolo Dei Tos ${ }^{2}$ \\ Giovanni Morana ${ }^{4}$ \\ Marco Massani ${ }^{3}$ \\ 'IV Department of Surgery, Regional \\ Hospital Treviso, Treviso, Italy; \\ ${ }^{2}$ Pathology Department, University of \\ Padova and Regional Hospital, Treviso, \\ Italy; ${ }^{3}$ II Department of Surgery, \\ Regional Hospital Treviso, Treviso, \\ Italy; ${ }^{4}$ Surgical Department, Regional \\ Hospital Treviso, Treviso, Italy
}

\begin{abstract}
Gastrointestinal stromal tumors (GISTs) are the most common mesenchymal tumors of the gastrointestinal tract. Significant prognostic heterogeneity has been described with GISTs, which can range from clinically benign to frankly malignant tumors. Although several GISTs classification systems have been established to identify tumors with high risk of relapse, there is a sample of patients who still do not receive an appropriate treatment. The classification scores by Fletcher et al and Miettinen and Lasota are the most widely clinically accepted, while the Memorial Sloan Kettering Cancer Center prognostic nomogram is considered the most feasible. There are several studies about new prognostic factors in radiological, biological, and surgical fields. Tumors with mixed growth pattern, enlarged vessels feeding, or draining the mass on computed tomography or with high standardized uptake values on positron emission tomography/ computed tomography should be considered as high-grade GISTs. Among biological markers, the most relevant are programmed cell death ligand 1, Pfetin, SETD2, SLITRK3, mir-215-5p, and monoglyceride lipase. These factors need to be further investigated in order to validate their use in risk stratification. Laparoscopy and open surgery can have the same oncological outcomes even for larger gastric tumors, up to $10 \mathrm{~cm}$. Laparoscopy should be considered the best surgical approach when executed by skilled surgeons and for tumor localized in reachable sites. Adjuvant chemotherapy with imatinib is recommended for high-grade GIST, but the optimal duration of this therapy is still debated. Some authors advocated that imatinib treatment should last for 5 years. A subgroup of GIST is represented by small GISTs, lesions $<2 \mathrm{~cm}$ in diameter, which need a radical surgical treatment in the presence of symptoms or when high-risk factors such as irregular borders, cystic spaces, ulceration, echogenic foci, internal heterogeneity, tumor progression during follow-up, small intestinal or colorectal localization are present.
\end{abstract}

Keywords: GIST, classification, prognostic factors, treatment, imatinib, small GIST

\section{Introduction}

Gastrointestinal stromal tumors (GISTs) are the most common mesenchymal tumors of the gastrointestinal tract $(80 \%)$, but they represent only $1 \%$ of all gastrointestinal tumors. Their incidence is around 1/1,00,000/year. ${ }^{1}$ The neoplastic GIST cells arise from a common precursor of the interstitial cells of Cajal in the normal myenteric plexus. ${ }^{2}$ The transformation of these cells is caused by activating mutations of KIT gene (about $75 \%)$ and less commonly PDGFRA (10\%-15\%). These genes encode receptor tyrosin kinases (TK), and their constitutive activation brings uncontrolled cell replication. Imatinib is a low-molecular weight TK inhibitor (TKI) that blocks the kinase activity of both KIT and PDGFRA. ${ }^{3}$ There is a small fraction of GIST, about $10 \%-15 \%$, which does not bear any mutation in these genes, so-called wild-type GIST. This group
Correspondence: Marco Massani

Ospedale Regionale, Piazza Ospedale I, 31 100 Treviso, Italy

Email marco.massani@aulss2.veneto.it 
is heterogeneous and includes two main subgroups: GIST characterized by inactivation of any of the components of the mitochondrial succinate dehydrogenase (SDH) complex which are essentially all gastric and NF1-inactivated GIST, mostly nongastric. Notably, SDH-inactivated GIST and NF1-inactivated GIST are typically associated with syndromic conditions, that is, Carney Stratakis syndrome and neurofibromatosis type 1 , respectively, but may also occur in an apparently sporadic context. ${ }^{4-7}$ Also belonging to the "wild-type" group are very rare GIST cases bearing activating mutations in BRAF, and GIST carrying gene fusions, for example, ETV6-NTRK3. ${ }^{8-10}$ In general, "wild-type" GISTs are poorly responsive to imatinib and may require alternative strategies, for example, sunitinib and regorafenib for SDH-inactivated GIST, dabrafenib for BRAF-mutated GIST, and inhibitors of TRK for GIST bearing ETV6-NTRK3. ${ }^{11,12}$ GISTs most commonly arise from the stomach (50\%-60\%) and small bowel $(30 \%-35 \%)$ and less frequently arise from the colon-rectum $(5 \%)$ and the esophagus $(<1 \%) .{ }^{13}$ They may also arise at extra gastrointestinal sites such as the omentum or mesentery. ${ }^{14}$ Significant prognostic heterogeneity has been described with GISTs, which can range from clinically benign to frankly malignant tumors. ${ }^{15,16}$ The standard treatment for localized primary GIST is complete surgical resection with clear margins. Nonetheless, the risk of recurrence remains even after complete resection. ${ }^{17}$ Imatinib is now recommended as adjuvant or supportive therapy in patients with high risk and advanced/unresectable disease by the European Society for Medical Oncology (ESMO) and National Comprehensive Cancer Network (NCCN) guidelines. They recommend the adjuvant use of TKI for patients with "a significant risk of relapse", particularly those with high-risk tumors, and also for tumors with intermediate risk up to 3 years. ${ }^{18,19}$ Therefore, there is a clinical need for a more reliable risk classification system that is simple to apply and able to stratify more precisely high-risk, low-risk, and very-low-risk patients for progression of disease. We focus on which is the most reliable classification in risk stratification and which could be the new prognostic factors.

\section{GISTs classification}

Several GISTs classification systems have been established to identify tumors with high grade of relapse, which may benefit from adjuvant chemotherapy. In 2002, Fletcher et $\mathrm{al}^{20}$ proposed the National Institute of Health (NIH) classification for defining the risk of aggressive behavior in GISTs. They divided GISTs into four groups with high, intermediate, low, and very low risk of progression, thereby excluding a benign category (Figure 1).

This classification is based on tumor size and mitotic count in 50 high power fields (HPF). ${ }^{20}$ In 2006, Miettinen et $\mathrm{al}^{15}$ analyzed 1,756 GISTs of the stomach and 906 GISTs of the small intestine in their work and published a new classification, also termed as the Armed Forces Institute of Pathology (AFIP) classification. Besides tumor size and mitotic count, this classification also takes into account the anatomic site of the primary tumor. Gastric GISTs show a much lower rate of aggressive behavior than comparable intestinal GISTs. Furthermore, they fixed a specific area of $5 \mathrm{~mm}^{2}$ for mitotic counting. Miettinen and Lasota established five risk groups, with eight subgroups, considering a benign class of tumor. ${ }^{15}$ The main difference between AFIP and NIH classifications is the importance given to the localization of the tumor. In 2007, Huang et al reevaluated NIH consensus criteria in 289 cases. They found no significant differences between the very low and the low risk groups, and they have been merged into a Level I risk group. Therefore, they included only GIST with size $>5 \mathrm{~cm}$ and $>10$ mitoses per $50 \mathrm{HPFs}$ into

\begin{tabular}{|l|l|l|}
\hline & Size & Mitotic count \\
\hline Very low risk & $<2 \mathrm{~cm}$ & $<5 / 50 \mathrm{HPF}$ \\
\hline Low risk & $2-5 \mathrm{~cm}$ & $<5 / 50 \mathrm{HPF}$ \\
\hline Intermediate risk & $<5 \mathrm{~cm}$ \\
$5-10 \mathrm{~cm}$ & $\begin{array}{l}6-10 / 50 \mathrm{HPF} \\
<5 / 50 \mathrm{HPF}\end{array}$ \\
\hline High risk & $>5 \mathrm{~cm}$ & $>5 / 50 \mathrm{HPF}$ \\
& $>10 \mathrm{~cm}$ & Any mitotic rate \\
& Any size & $>10$
\end{tabular}

Figure I NIH classification.

Note: Adapted from Hum Pathol, 33(5), Fletcher CD, Berman JJ, Corless C, et al, Diagnosis of gastrointestinal stromal tumors: A consensus approach, 459-465, Copyright 2002, with permission from Elsevier. ${ }^{20}$

Abbreviations: HPF, high power field; NIH, National Institute of Health. 
Level IV, because of prognostic heterogeneity in the high-risk category of NIH scheme. ${ }^{16}$ In 2008 , Joensuu et $\mathrm{al}^{3}$ proposed the "modified NIH classification" in which they introduced the tumor rupture as a prognostic factor (Figure 2).

Furthermore, they suggested a new modified high-risk group that would include all patients designated as high risk by the NIH classification and, in addition, patients with nongastric tumors $2-5 \mathrm{~cm}$ and $>5$ mitoses per 50 HPFs, or $5-10 \mathrm{~cm}$ and $\leq 5$ mitoses per $50 \mathrm{HPFs}$, and all patients with tumor rupture into the abdominal cavity regardless of tumor size or mitotic count. ${ }^{3}$ In 2009, the Memorial Sloan Kettering Cancer Center (MSKCC) developed a prognostic nomogram: it is based on three factors score (size, site, and mitotic index [MI]), and the sum of the three scores corresponds to a prediction of 2- and 5-year recurrence-free survival (RFS) after surgical resection of a localized primary GIST $^{21}$ (Figure 3).

A survival nomogram has been subsequently generated: it considers site, size, and MI as continuous variables, providing estimates stratified for patients aged $\leq 65$ and $>65$ years. ${ }^{22}$ Bischof et al, in 2014, proposed a nomogram to predict disease-free survival (DFS) following surgical resection of GIST. They considered sex, tumor size, tumor site, and mitotic rate as prognostic factors. This nomogram stratified patients into prognostic groups and performed well on internal validation. ${ }^{23}$ In 2010, the first TNM classification for GIST was published. It was based on the classification of Miettinen et al, ${ }^{15}$ but it also considered the mitotic rate.
There are eight stages corresponding to the eight subgroups of the AFIP classification (Figure 4).

However, ESMO guidelines do not recommend the use of this classification. Then, which is the most reliable and most easily used classification? Clinically, the classification score by Fletcher et al and Miettinen and Lasota ${ }^{20,15}$ are the most widely accepted. Several authors and the current ESMO guidelines have recommended the standardization of mitotic counting for GIST to avoid upgrading of patients' risk. Schmieder et $\mathrm{l}^{24}$ reevaluated the impact of five widely applied and well established GIST risk classification systems (scores by Fletcher, Miettinen, Huang, Joensuu, and TNM classification) on a series of 558 GIST patients with longterm follow-up after $\mathrm{R} 0$ resection. This is a multicenter study encompassing 18 oncological centers in South Germany, from 2006 to 2012. Patients diagnosed before 2006 were registered retrospectively. After 2006, patients were recorded in a prospective manner. The results did not show any significant differences in terms of disease relapse prediction by all the scores, so they appeared to be equivalent. Anyway, the subgroup of high-risk patients was heterogeneous because of the different disease pathogenesis with consequences for tumor progression and clinical outcome. ${ }^{24}$ Heterogeneity in GISTs might not only be influenced by molecular, but also by other nongenetic factors (age, gender, tumor site, and syndromic occurrence), and maybe these factors should be considered in a prognostic score. Of course, standardization of mitotic count remains a basic principle for the score

\begin{tabular}{|l|l|l|l|}
\hline Risk category & $\begin{array}{l}\text { Tumor size } \\
(\mathrm{cm})\end{array}$ & $\begin{array}{l}\text { Mitotic index } \\
(\text { per } 50 \text { HPFs })\end{array}$ & $\begin{array}{l}\text { Primary } \\
\text { tumor site }\end{array}$ \\
\hline Very low risk & $<2.0$ & $\leq 5$ & Any \\
\hline Low risk & $2.1-5.0$ & $\leq 5$ & Any \\
\hline Intermediate risk & $2.1-5.0$ & $>5$ & Gastric \\
& $<5.0$ & $6-10$ & Any \\
& $5.1-10.0$ & $\leq 5$ & Gastric \\
\hline Any & $>10.0$ & Any & Tumor pressure \\
\hline Angh risk & Any & $>10$ & Any \\
& $>5.0$ & $>5$ & Any \\
& $2.1-5.0$ & $>5$ & Any \\
\hline $5.1-10.0$ & $\leq 5$ & Nongastric \\
& & & Nongastric \\
\hline
\end{tabular}

Figure 2 Modified NIH classification.

Notes: Adapted from Hum Pathol, 39, Joensuu, Risk stratification of patients diagnosed with gastrointestinal stromal tumor, I4II-1419, Copyright 2008, with permission from Elsevier. ${ }^{3}$

Abbreviations: HPF, high power field; NIH, National Institute of Health. 


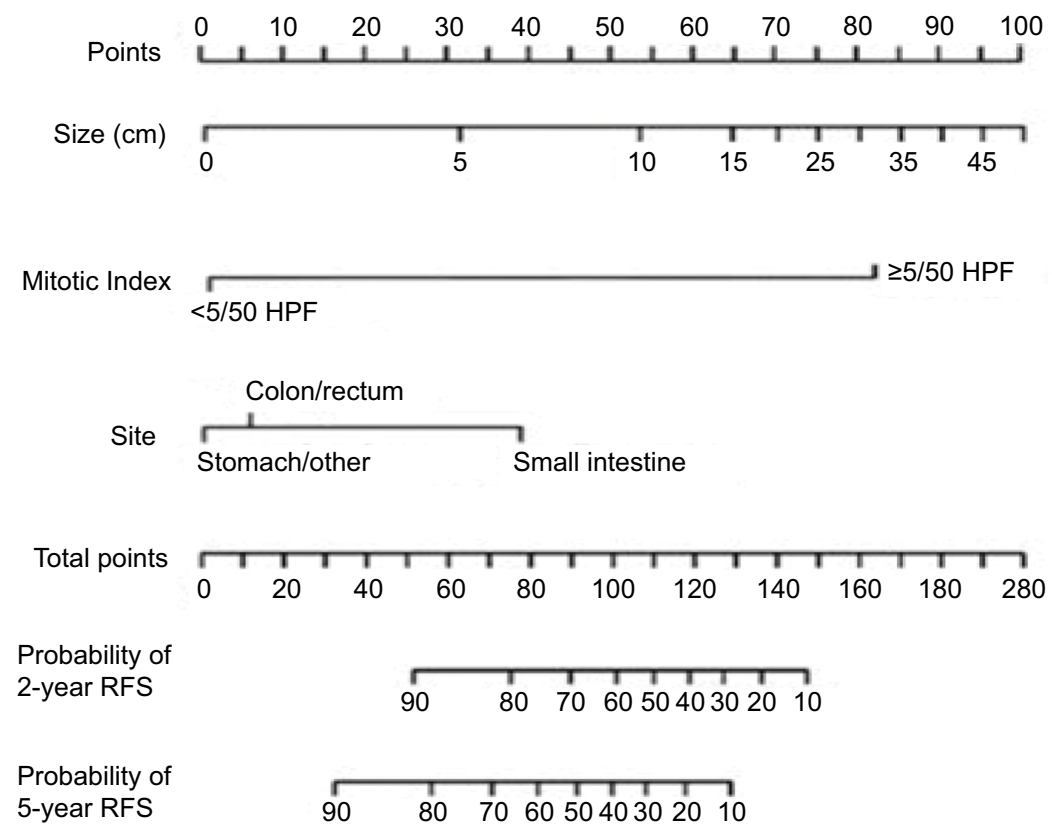

Figure 3 MSKCC prognostic nomogram.

Note: Adapted from Lancet Oncol, IO(II), Gold JS, Gönen M, Gutiérrez A, et al, Development and validation of a prognostic nomogram for recurrence-free survival after complete surgical resection of localised primary gastrointestinal stromal tumour: a retrospective analysis, 1045-1052, Copyright 2009, with permission from Elsevier. ${ }^{21}$ Abbreviations: HPF, high power field; MSKCC, Memorial Sloan Kettering Cancer Center.

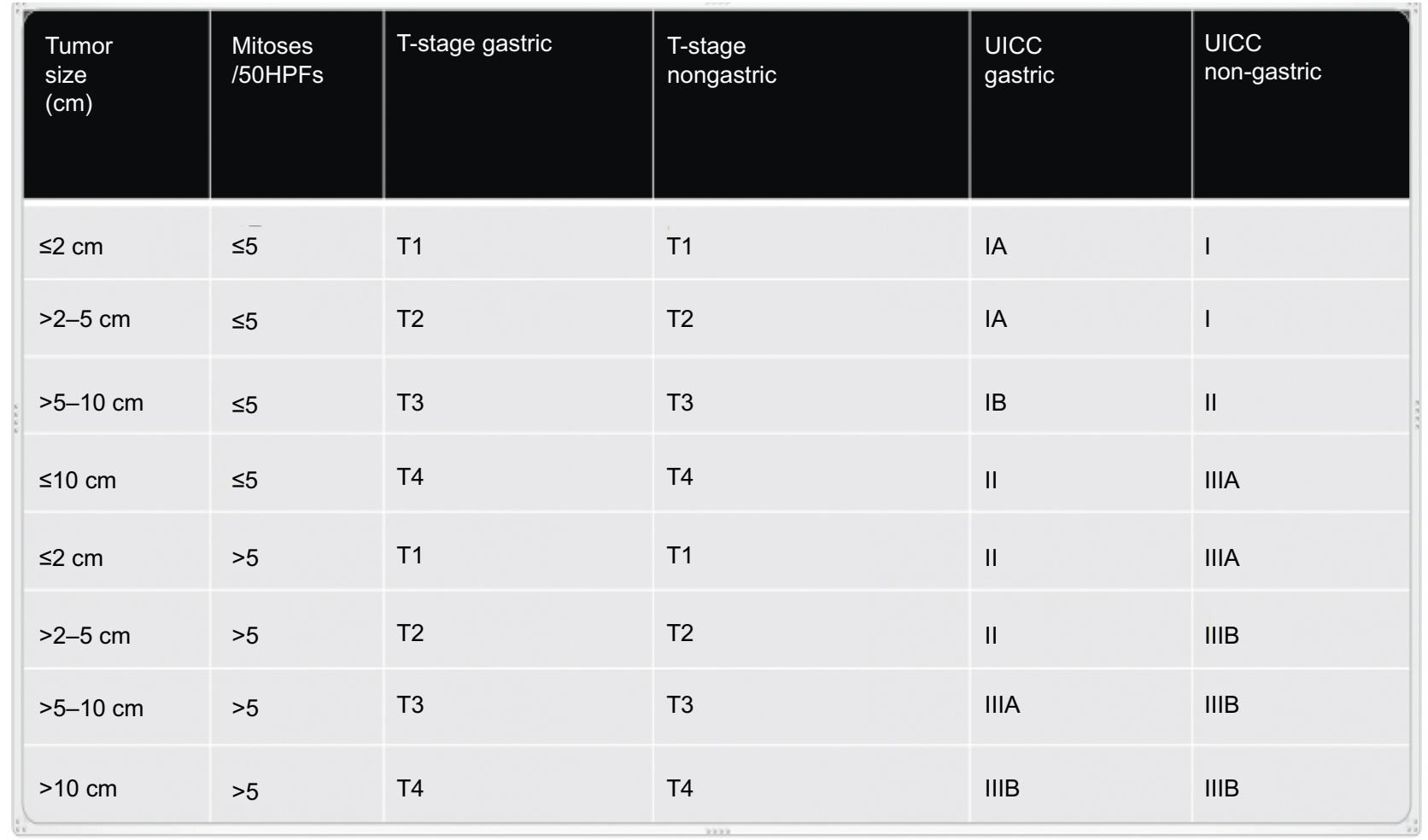

Figure 4 TNM classification for GIST. Adapted from Agaimy A., Gastrointestinal stromal tumor (GIST) from risk stratification systems to the new TNM proposal: more questions than answers? A review emphasizing the need for a standardized GIST reporting. Int J Clin Exp Pathol. 2010;3(5):46I-47I. ${ }^{67}$

Abbreviations: GIST, gastrointestinal stromal tumor; HPF, high power field; UICC, Union for International Cancer Control.

quality. Many authors consider the MSKCC prognostic nomogram more feasible in clinical practice than the other classifications. Chok et al tried to validate the MSKCC nomogram and to compare its predictive accuracy against other established risk classification systems, including the NIH, AFIP, and Joensuu criteria. They published a singlecohort retrospective study of 289 patients who underwent surgical resection for primary localized GISTs without 
adjuvant imatinib therapy and compared the actuarial RFS with the predicted RFS. The MSKCC nomogram and AFIP criteria had the best predictive accuracy for tumor recurrence compared with the NIH and Joensuu risk classification systems. However, the MSKCC nomogram slightly underestimated the probability of RFS after surgical resection of GISTs. ${ }^{17}$ Belfiori et al also compared MSKCC to NIH, NIH modified, and AFIP risk classifications. They confirmed the superiority of nomogram with respect to the other scores, even though it was not impeccable in predicting the RFS. Furthermore, they remarked that the main limitation of MSKCC nomogram remains the nonlinear consideration of mitotic count. ${ }^{25}$ Notably, the conventional clinicopathological parameters for risk assessment are poorly predictive of the outcome when dealing with "wild-type" GIST. In fact, SDH-inactivated GIST tends to have an indolent clinical behavior even in the advanced setting. ${ }^{26}$ Localized NF1associated GISTs usually have a prolonged course but tend to become clinically aggressive once metastatic. ${ }^{27}$

\section{High-grade GISTs}

Current guidelines recommend adjuvant therapy with imatinib in high-grade GISTs. Many classifications are useful to define risk stratification at the time of diagnosis, and it is very important to establish future treatment. High-risk GISTs are tumors that have a high number of mitosis, increase in size, and do not come from the stomach. Another important prognostic factor is tumor rupture. This is a very heterogeneous class of tumors, and for this reason several authors try to identify new prognostic factors. The biological field seems to be the most widely undertaken path in the literature. Radiological imaging could be another interesting field to analyze. Laparoscopic and open approaches are compared to verify different oncological outcomes. Even between small GISTs (tumors $<2 \mathrm{~cm}$ in diameter), there are high-risk factors that should be carefully evaluated.

\section{Imaging}

None of the many validated GISTs' classification included any radiological findings as a prognostic factor, although there are some studies that have identified new risk factors. Zhou et $\mathrm{al}^{28}$ analyzed some computed tomography's features of 129 patients with GIST $>2 \mathrm{~cm}$ : primary tumor location, size, margin, shape, density, calcification, growth patterns, enhancement pattern, degree of enhancement, enlarged vessels feeding or draining the mass (EVFDM), necrosis, direct organ invasion, and lymphadenopathy. All these features were associated with the risk stratifications, as determined by univariate analysis. Only lesion size, growth pattern, and EVFDM remained independent risk factors in multinomial logistic regression analysis. The tumors with size $>10 \mathrm{~cm}$ or 5-10 $\mathrm{cm}$ mixed growth pattern, or EVFDM were likely to be higher risk GISTs than those with size $<5 \mathrm{~cm}$, endoluminal growth pattern, or without EVFDM. ${ }^{28}$ Miyake et al assessed the potential value of preoperative 18F-FDG positron emission tomography to predict postoperative recurrence of solitary localized primary GIST after radical resection. Ring-shaped uptake and intense uptake were significantly associated with Joensuu high risk. Univariate analysis showed that ring-shaped uptake, intense uptake, size $>5 \mathrm{~cm}$, and Joensuu high risk were significantly associated with inferior RFS. Multivariate analysis showed that ringshaped uptake $(P=0.004)$ and Joensuu high risk $(P=0.021)$ were independent adverse prognostic factors of postoperative recurrence. ${ }^{29}$ Another important tool for clinical evaluation of GIST is positron emission tomography/computed tomography. It facilitates both anatomic and functional evaluation of tumors and has become the standard imaging for GIST. Tokumoto et al determined the correlation of the risk category with standardized uptake values (SUV) max, tumor size, mitotic count, and MIB-1 index. The cutoff value for SUV max was found to be 3.0 between the low-risk and high-risk malignancy groups. Using univariate analysis in the highrisk malignancy group they determined that the SUV max value, mitotic count, and the MIB-1 index, but not tumor size, were predictive risk factors of malignancy. In a multivariate analysis SUV max was the only predictive risk factor for the high-risk malignancy group. This study recommended that submucosal gastric tumors with an SUV max $>3.0$ must be resected, even if the tumor size is $<2 \mathrm{~cm}$, because the tumors may have a high malignant potential. ${ }^{30}$

\section{Biological markers}

High-grade GIST group is a heterogeneous class. Therefore, many studies tried to identify biological markers as new prognostic factors. In particular, the aim was to identify prognostic markers that are able to select the best group of patients for adjuvant imatinib therapy. Many biological factors have been investigated. These factors are often related to high-grade GIST, but their prognostic value and their role to guide imatinib therapy are still debated (Table 1).

- The programmed cell death 1 (PD1)/programmed cell death ligand 1 (PDL1) pathway is a key inhibitor of the immune response. PDL1 expression was higher in AFIP low-risk samples than in high-risk samples. PDL1 expression was also higher in samples without metastatic 
Table I Biological markers

\begin{tabular}{ll}
\hline Markers & Prognosis \\
\hline PDLI & + \\
Pfetin & + \\
SETD2 & - \\
SLITRK3 & - \\
miR-2I5-5 P & $+1-*$ \\
MGLL & - \\
\hline
\end{tabular}

Note: *Prognostic significance is still controversial.

Abbreviations: +, favorable prognosis; -, poor prognosis; MGLL, monoglyceride lipase; PDLI, programmed cell death ligand I.

relapse than in samples with metastatic relapse, suggesting favorable prognostic value. ${ }^{31}$

- Pfetin, a potassium channel protein, is a prognostic biomarker for GIST. ${ }^{32}$ Pfetin is an independent predictor of recurrence/metastasis for completely resected primary, localized GIST. There is an inverse relationship between Pfetin expression and risk of recurrence. ${ }^{33}$

- SETD2 may represent a novel GIST tumor suppressor gene, which contributes to GIST progression. SETD2 mutations are exclusively found in patients with highrisk/metastatic GISTs with a prevalence rate of approximately $11 \%$ but not in patients with low/intermediate risk. SETD2 mutations may occur at a later stage of tumorigenesis and appear to be associated with GIST progression, rather than initiation. Patients with GIST with SETD2 mutations or DNA methylation phenotypes showed shorter relapse-free survival. ${ }^{34}$

- SLITRK3 is one of the six isoforms of SLIT and neurotrophic tyrosine receptor kinase (NTRK)-like family member (Slitrk1-6), which are neuronal transmembrane proteins that control neurite growth. GISTs may originate from the interstitial cells of Cajal, with pacemaker potentials suggesting that mutations in genes involved in synapse or neural development may underlie GIST behavior. Increasing SLITRK3 expression correlates with decreased overall survival (OS) and DFS. SLITRK3 mRNA expression level increased according to NIH risk classification. SLITRK3 protein level is closely associated with tumor site, tumor size, and MI. Patients with high SLITRK3 expression, especially those who are also in the NIH high-risk groups, should receive IM adjuvant therapy and close follow-up management after surgery. ${ }^{35}$

- Dysregulation of miRNAs has been observed virtually in all major types of cancer, whereas the miRNA signature in GIST is not well characterized yet. miR-215-5 $\mathrm{p}$ is negatively correlated with the risk grade of GIST. The identification of miRNA profile GIST would be significant for further identification of new targets and development of novel therapeutics. ${ }^{36}$

- Monoglyceride lipase (MGLL) is a lipid metabolic enzyme causatively implicated in GIST progression. MGLL overexpression is associated with adverse clinicopathological factors and is independently predictive of unfavorable prognosis, suggesting its causative role in conferring aggressive phenotypes to primary, localized, imatinib-naïve GISTs. ${ }^{37}$

- Several lines of observations suggest that KIT/PDGFRA mutational status also impacts on GIST natural course, with most of PDGFRA-mutated GIST showing a more favorable outcome and GIST with a structural variant in the proximal region of KIT exon 11, for example, W557_K558del, or with exon 9 KIT mutation, that is, A502_Y503dup, behaving more aggressively. ${ }^{38,39}$

There are other biological factors that are currently used in the diagnosis of GISTs, whose prognostic role could be investigated, since only initial studies have been published at the moment. Some of them are as follows: phosphorylated form of histone 3 (PHH3), DOG-1, ETV1, and Ki 67. Immunohistochemistry for the $\mathrm{PHH} 3$, which is present during early prophase, has been shown to be a reliable mitosis-specific marker. There is a tendency to undergrade GISTs based on H\&E compared with $\mathrm{PHH} 3$, which alters the stage, risk of disease progression, and treatment recommendations. $\mathrm{PHH} 3$ cutoff value of seven mitoses or higher was associated with worse OS. ${ }^{40}$ DOG1 (discovered on GIST-1) shows a higher sensitivity as a diagnostic marker than KIT. Patients with a strong DOG1 expression, tumor size $\geq 5 \mathrm{~cm}$, and mutations of KIT or PDGFRA had a worse prognosis. On the contrary, DOG1-negative patients have a more favorable postoperative 2-year RFS rate. ${ }^{41}$ ETV1, a member of the ETS family, is upregulated in GISTs, and its signaling is integrated into a cellular signaling network for resistance to apoptosis, tumor cell invasion, and survival. ETV1 was strongly expressed in the GISTs, which can aid in the diagnosis of GISTs particularly when KIT is negative in the high-risk group. The prognostic significance is still controversial. ${ }^{42} \mathrm{Ki} 67$ is a good marker of cell proliferation in a variety of tumors. There is a greater rate of Ki67 overexpression in NIH-intermediate and NIH-high GIST risk groups. Ki67 expression may be an effective complement to the NIH criteria for predicting the risk of malignant GIST, especially for intermediate- and high-risk cases. ${ }^{43}$ Cancer-associated inflammation is shown to promote tumor progression and metastases via suppressing antitumor immunity, and it is closely related to various stages of tumor development. There is a significant association 
between low hemoglobin $(\mathrm{Hb})$ levels, an elevated white blood cell (WBC) count, neutrophil/lymphocyte ratio (NLR), derived NLR (dNLR), and decreased OS. Low hemoglobin level, an elevated WBC, as well as an elevated NLR, dNLR, and platelet/lymphocyte ratio (PLR) are parameters that were significantly associated with poor RFS. Of all the studied variables, only elevated dNLR was associated with a higher risk of local or distant recurrence after adjusting for Miettinen score. The association between NLR and PLR with disease progression was independent of the Miettinen score, but the inclusion of these variables did not improve the clinical risk prediction of 2-year disease progression and death. This finding indicates that NLR and PLR are independent prognostic variables for RFS, but their impact on improving prognostication in surgical-treated GIST patients appears to be limited. ${ }^{44}$ However, in patients receiving adjuvant imatinib, peripheral blood counts did not show any prognostic significance. Preoperative NLR is a feasible and reproducible peripheral biomarker that helps identify patients for intensive adjuvant therapy and frequent surveillance.$^{45}$ Molecular stratification may support the clinician in decision-making for adjuvant therapy, particularly for intermediate-risk GIST, where the presence of unfavorable mutations may tip the scales on the side of adjuvant therapy.

\section{Surgery}

Surgery is the main treatment for primary GIST with no evidence of metastasis. The standard is complete surgical excision, avoiding tumor rupture, with no dissection of clinically negative lymph nodes. ${ }^{18}$ Nowadays, laparoscopy is increasingly used in surgery for its well-known advantages: earlier patient recovery, reduction of hospitalization, and better esthetic/cosmetic results. Laparoscopic approach is more often applied to treat GIST, and its feasibility has been demonstrated in several studies. This technique must also respect the principles of oncological radicality, in particular, avoiding the rupture of the tumor mass. For this reason, both NCCN and ESMO guidelines recommend an open approach in case of large GIST $(>5 \mathrm{~cm})$ or if the tumor is difficult to access. On the other hand, for gastric GIST, there are several groups that have gone beyond the size limit imposed by the guidelines to verify the consequences also in prognostic terms. For these tumors, a wedge resection should be performed, when it is possible. Hsiao et al showed that the laparoscopic approach is feasible for tumors up to $8 \mathrm{~cm}$ in diameter with an acceptable operative and oncological outcome after an intermediate follow-up. However, they emphasize how the gastric location of the tumor is an important factor to be evaluated, considering some gastric sites such as the cardias, the small gastric curve, or prepyloric area could be more challenging for a laparoscopic resection. ${ }^{46}$ Lin et al analyzed 23 patients with tumor $>5 \mathrm{~cm}$ in diameter treated by laparoscopic resection and were randomly matched $(1: 1)$ by tumor size $( \pm 1 \mathrm{~cm})$ to patients with open resection. They demonstrated that laparoscopic resection of gastric GISTs up to $10 \mathrm{~cm}$ results in operative durations, morbidity, and outcomes that are similar to those of open resection but is associated with shorter hospital stays, and that tumor location is clearly an important factor in the selection of an operative approach. They suggested a neoadjuvant treatment with imatinib to prevent the risks of rupture or bleeding of the tumor during the resection. ${ }^{47}$ Piessen et al showed that overall morbidity, surgical morbidity, and medical morbidity were significantly lower in laparoscopic group. Five-year RFS was also significantly better in this group. Patients with gastric GISTs $>5 \mathrm{~cm}$ had hospital morbidity and 5-year RFS similar to the open group. ${ }^{48}$

In conclusion, laparoscopy and open surgery can have the same oncological outcomes even for larger gastric tumors, up to $10 \mathrm{~cm}$. Laparoscopy should be considered the best surgical approach when executed by skilled surgeons and for tumors localized in reachable sites. Obviously, radicality must be respected: when the rupture or the bleeding of the mass becomes an issue, an open approach should be advocated to prevent any spillage of the tumor.

\section{Imatinib}

Imatinib, a TKI, is now recommended as an adjuvant or supportive therapy in patients with high risk and advanced/ unresectable disease. There are many prognostic score systems to evaluate recurrence risk. The NCCN considers prognostic factors as tumor size, mitotic rate, and tumor site. Joensuu et al developed two risk stratification scores to predict GIST recurrence in patients treated with adjuvant imatinib therapy. ${ }^{49} \mathrm{~A}$ risk score was generated using five independent factors associated with favorable RFS: low tumor mitotic count (with central assessment), location in the stomach, adjuvant imatinib for 36 months, small tumor size, and absence of rupture. Another risk score included two factors that were most strongly associated with RFS: tumor mitotic count assessed centrally and tumor site. Both scores generated were effective in stratifying the risk of GIST recurrence in patient populations treated with adjuvant imatinib. GISTs with high mitotic count arising at nongastric sites recur frequently despite adjuvant imatinib, and some of such tumors recur even when the patient is on imatinib suggesting that more efficient treatments need to be pursued. Despite guidelines recommendations and the various prognostic scores, there is a sample of patients who still do 
not receive an appropriate treatment. Bischof et al considered all patients who underwent surgery for primary GIST in USA and Canada between January 2009 and December 2012. They found that following surgery, $23 \%$ of patients were undertreated and $2 \%$ of patients were overtreated, when compared with NCCN guidelines. They tried to identify the clinicopathologic predictors of treatment with adjuvant imatinib following surgical resection of GIST. Tumor size, mitotic rate, and neoadjuvant imatinib therapy were associated with receipt of adjuvant imatinib therapy. Interestingly, tumor site was not associated with receipt of adjuvant imatinib therapy. The reasons for undertreatment of these patients remain unclear. ${ }^{50}$ Guérin et al attempted to determine the extent of physician underestimation of risk of recurrence after complete primary GIST resection. Findings suggest that borderline measures were more difficult to categorize, and some physicians might not adequately take into account tumor location. High-risk tumors of intermediate-level tumor size, intermediate-level mitotic count, and nongastric GIST location were frequently underestimated. High-risk patients were more likely to have a planned adjuvant therapy duration of $<3$ years after resection if their recurrence risk was underestimated compared with patients whose risk was not underestimated. Planned adjuvant therapy duration of $<3$ years was associated with reduced RFS. ${ }^{51}$ Another important problem is about duration of adjuvant imatinib therapy. In the ACOSOG Z9001 study, Dematteo et al demonstrated that $400 \mathrm{mg}$ of daily imatinib for 1 year following surgery improved RFS in patients with GIST at least $3 \mathrm{~cm}$ in size compared with placebo alone. ${ }^{52}$ In the SSGXVIII/AIO trial, Joensuu et al found that 3 years of adjuvant imatinib was associated with improved RFS and OS compared with 1 year of adjuvant imatinib in patients with high-risk GIST (tumor size $>10 \mathrm{~cm}$, mitotic rate $>10$ mitoses per $50 \mathrm{HPF}$, tumor diameter $>5 \mathrm{~cm}$, and mitotic rate $>5$ mitoses/50 HPF, or tumor rupture). ${ }^{53}$ Currently, ESMO and NCCN guidelines recommend 3 years of adjuvant imatinib for high-risk GIST. ${ }^{18,19}$ In their review, Trent and Subramanian confirmed that adjuvant imatinib therapy for at least 3 years can be recommended for patients who have an intermediate or high risk of GIST recurrence. ${ }^{54}$ However, the optimal duration of therapy, and whether high-risk patients should use adjuvant imatinib continuously, remain unknown. Risk of recurrence should be assessed using accepted risk assessment criteria, based on tumor size and MI, to appropriately identify which patients are at greatest risk and are most likely to benefit from adjuvant imatinib therapy following surgical resection. Other factors such as GISTs mutational status, tumor location, and tumor rupture can add prognostic value. Lin et al suggest that patients with high-risk GIST should receive imatinib treatment for at least 5 years following surgical resection. They observed how the recurrence rate increased when imatinib treatment was stopped, arriving almost at the same rate of patients who did not receive any therapy. So, the question is does adjuvant imatinib therapy only delay the patients' relapse? The result showed that the RFS of patients with high-risk GIST increased significantly due to the prolongation of medication time. ${ }^{55}$ In their intergroup randomized trial of adjuvant imatinib vs no further therapy after surgery, Casali et al analyzed imatinib failure-free survival (IFFS). They found that the trend of IFFS in the adjuvant arm at least suggested that exposure to imatinib in the adjuvant setting did not induce a selection pressure toward secondary resistance, at least within the time interval that adjuvant imatinib was administered in this study (2 years). A crucial finding is that adjuvant imatinib therapy does not seem to cure minimum residual disease in patients with resected GIST. In fact, they observed how stopping adjuvant therapy is followed by relapse in at least most patients expected to experience relapse in the absence of any adjuvant therapy ${ }^{56}$ Of course, the most logical consequence for clinical research is the attempt to prolong treatment duration. Other trials are necessary to rule out a detrimental effect of longlasting adjuvant treatment in terms of an earlier incidence of secondary resistance. Maybe, new strategies of administering targeted therapies will be more effective possibly guided by molecular biomarkers during treatment. In a recent study, Joensuu found a correlation between the duration of adjuvant imatinib and KIT deletion mutations. The adverse prognostic influence of some frequent mutations, such as deletion mutations that involve KIT exon 11 codons 557 and/or 558, was no longer detectable in patients treated with adjuvant imatinib for 3 years. Tumor mutation analysis aids in the selection of patients for adjuvant imatinib treatment because patients with KIT deletion mutations benefit more from 3 years of adjuvant imatinib than patients with other mutations. ${ }^{57}$

\section{Small GIST}

GISTs $<2 \mathrm{~cm}$ are defined as small GISTs. Often, they are incidental findings during procedures for other diseases. Based on the AFIP risk criteria, there is a "very low, if any malignant potential" for all gastric tumors $<2 \mathrm{~cm}$ with an $\mathrm{MI}<5$, while "high malignant potential" is attributed to all rectal GISTs with an MI >5, irrespective of the size. The category of small GIST includes the so-called microGIST, which are lesions with a size $\leq 1 \mathrm{~cm}$, considered mostly self-limiting and with no malignant potential. ${ }^{58,59}$ With the exception of microGIST, the natural course of small GIST in general is not well defined. Compared with larger GISTs, GISTs $<2 \mathrm{~cm}$ have a lower frequency of mutations overall and particularly of KIT exon 11 mutations, 
while carrying a higher fraction of rare/novel mutations, likely of low pathogenic potential. ${ }^{60}$ However, most of the data on small GIST are limited to pathological analyses and autopsy studies. Coe et al published the first population-based analysis of malignant GIST $<2 \mathrm{~cm}$ throughout the gastrointestinal (GI) tract and identified a mortality risk that has been underappreciated by gastroenterologists, surgeons, and oncologists. ${ }^{61}$ In fact, they found that the 5-year GIST-specific mortality rate was $12.9 \%$. Furthermore, $11 \%$ of patients with GIST $<2 \mathrm{~cm}$ had regional or distant metastases. However, this study does not include undiagnosed or asymptomatic tumors. Therefore, the described rates are specific to small GISTs that are coded as malignant and have been histologically evaluated following biopsy or resection, likely overestimating the GIST-specific mortality of all small GISTs. Furthermore, no information on the molecular characteristics of the cases is provided, and possibly the series includes cases that are pathogenetically heterogeneous. The treatment of these tumors is still debated.
Surgery could be an overtreatment, especially in elderly patients with gastric small GISTs. On the other hand, there is a risk of underestimating these tumors. The NCCN recommends resection for all GISTs $\geq 20 \mathrm{~mm}$; no clear statement is made regarding incidentally encountered small GISTs $(<20 \mathrm{~mm})$ due to the insufficient data. Complete surgical resection is suggested for those gastric GISTs having high-risk endoscopic ultrasonography (EUS) features, and endoscopic surveillance at 6-12 months intervals for the lesions without high-risk features. ${ }^{19}$ ESMO guidelines suggest control at short term (eg, at 3 months) and then to prolong the follow-up intervals in case of no growth for small lesions, if follow-up strategy is the preferred choice. ${ }^{18}$ Japanese guidelines state that lesions $<20 \mathrm{~mm}$ in size and without ulceration or surface depression can be followed up endoscopically once or twice a year. ${ }^{62}$ In a recent review, Nishida et $\mathrm{al}^{63}$ proposed an algorithm to guide diagnostic and treatment strategies for patients with small GISTs (Figure 5).

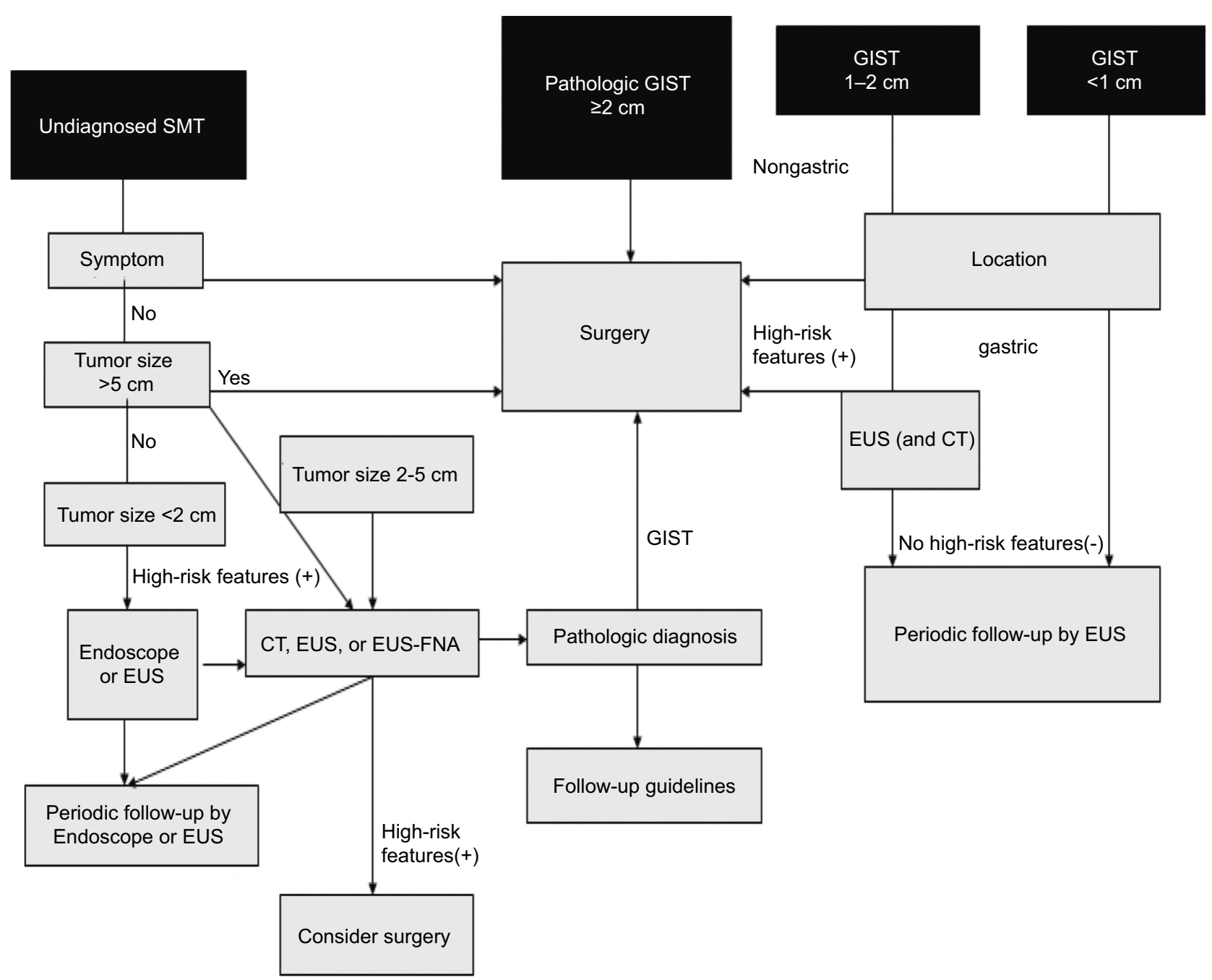

Figure 5 Small GIST treatment.

Note: Adapted from Nishida T et al, Diagnostic and treatment strategy for small gastrointestinal stromal tumors, Cancer, 2016,122(20), 31 I0-3।I8. ${ }^{63}$

Abbreviations: EUS, endoscopic ultrasonography; EUS-FNA, EUS-guided fine-needle aspiration; GIST, gastrointestinal stromal tumor; SMT, submucosal tumor. 
They considered the following as high-risk features: the presence of symptoms, irregular borders, cystic spaces, ulceration, echogenic foci, internal heterogeneity, tumor progression during follow-up, small intestinal or colorectal localization. ${ }^{63}$ EUS surveillance is recommended to measure the exact dimensions and to provide information on any change to high-risk sonographic features related to the increased likelihood of malignant behavior. These high-risk EUS features suspicious of malignancy for GISTs were defined as larger size, irregular borders, heterogeneous echo patterns, presence of anechoic spaces, and echogenic foci..$^{64,65}$ In most cases, EUS findings allow only a presumptive diagnosis and determine the need for further explorations such as tissue sampling, surgery, or follow-up. A tissue diagnosis with EUS-guided fine-needle aspiration (EUS-FNA) is generally performed, but it provides inadequate material in up to $33.3 \%$ of the cases. Even in the case of a GIST diagnosis accomplished by EUS-FNA, evaluation of the malignant potential of the tumor based on the MI may not be possible due to the lack of sufficient material as required for prompt investigation. ${ }^{66}$ Therefore, even for the small GIST, the site of origin is an important prognostic factor. Other risk factors are the presence of symptoms, ultrasound features, and growth during follow-up.

\section{Conclusion}

Among the current classifications, many authors consider the MSKCC prognostic nomogram more feasible in clinical practice than the other classifications, even though it was not faultless in predicting the RFS. Furthermore, the main limitation of MSKCC nomogram remains the nonlinear consideration of mitotic count. Laparoscopy should not be considered as a negative prognostic factor, if oncological radicality has been respected. A good staging is also important in small GIST to avoid over/undertreatment. EUS seems to be the most valid tool for the correct characterization of these tumors. Imatinib therapy is recommended in high-grade GIST. Some studies suggest that adjuvant imatinib therapy should be prolonged for at least 5 years in high-grade GISTs, because of prolonged RFS. Probably, risk scores should be enriched with other factors such as radiological signs and biological markers. There are several studies about new prognostic factors especially in biological field. These factors need to be further investigated in order to validate their use in risk stratification. The genetic landscape of GIST appears to be very heterogeneous; a deeper understanding of the molecular mechanism underlying GIST progression would hopefully improve risk assessment. What we can hope is a new prognostic classification based only on biological markers, which could be more reliable than current classifications.

\section{Disclosure}

The authors report no conflicts of interest in this work.

\section{References}

1. Nilsson B, Bumming P, Meis-Kindblom JM, et al. Gastrointestinal stromal tumors: the incidence, prevalence clinical course, and prognostication in the pre-imatinib mesylate era-a population based study in western Sweden. Cancer. 2005;103:821-829.

2. Nishida T, Hirota S. Biological and clinical review of stromal tumors in the gastrointestinal tract. Histol Histopathol. 2000;15: 1293-1301.

3. Joensuu H. Risk stratification of patients diagnosed with gastrointestinal stromal tumor. Hum Pathol. 2008;39:1411-1419.

4. McWhinney SR, Pasini B, Stratakis CA; International Carney Triad and Carney-Stratakis Syndrome Consortium. Familial gastrointestinal stromal tumors and germ-line mutations. $N$ Engl J Med. 2007;357(10):1054-1056.

5. Pasini B, Mcwhinney SR, Bei T, et al. Clinical and molecular genetics of patients with the Carney-Stratakis syndrome and germline mutations of the genes coding for the succinate dehydrogenase subunits SDHB, SDHC, and SDHD. Eur J Hum Genet. 2008;16(1):79-88.

6. Gasparotto D, Rossi S, Polano M, et al. Quadruple-negative GIST is a sentinel for unrecognized neurofibromatosis type 1 syndrome. Clin Cancer Res. 2017;23:273-282.

7. Rossi S, Gasparotto D, Cacciatore M, et al. Neurofibromin C terminusspecific antibody (clone NFC) is a valuable tool for the identification of NF1-inactivated GISTs. Mod Pathol. 2018;31(1):160-168.

8. Agaram NP, Wong GC, Guo T, et al. Novel V600E BRAF mutations in imatinib-naive and imatinib-resistant gastrointestinal stromal tumors. Gen Chrom Cancer. 2008;47:853-859.

9. Brenca M, Rossi S, Polano M, et al. Transcriptome sequencing identifies ETV6-NTRK3 as a gene fusion involved in GIST. $J$ Pathol. 2016;238(4):543-549.

10. Shi E, Chmielecki J, Tang CM, et al. FGFR1 and NTRK3 actionable alterations in "Wild-Type" gastrointestinal stromal tumors. J Transl Med. 2016;14(1):339.

11. Florou V, Wilky BA, Trent JC. Latest advances in adult gastrointestinal stromal tumors. Future Oncol. 2017;13(24):2183-2193.

12. Wong NA. Gastrointestinal stromal tumours-an update for histopathologists. Histopathology. 2011;59(5):807-821.

13. Woodall CE, Brock GN, Fan J, et al. An evaluation of 2537 gastrointestinal stromal tumors for a proposed clinical staging system. Arch Surg. 2009;144(7):670-678.

14. Demetri GD, Benjamin RS, Blanke CD, et al. NCCN Task Force report: management of patients with gastrointestinal stromal tumor (GIST)update of the NCCN Clinical Practice Guidelines. J Natl Compr Canc Netw. 2007;5(Suppl 2):S1-S29.

15. Miettinen M, Lasota J. Gastrointestinal stromal tumors: pathology and prognosis at different sites. Semin Diagn Pathol. 2006;23:70-83.

16. Huang HY, Li CF, Huang WW, et al. A modification of NIH consensus criteria to better distinguish the highly lethal subset of primary localized gastrointestinal stromal tumors: a subdivision of the original high-risk group on the basis of outcome. Surgery. 2007;141(6): 748-756.

17. Chok AY, Goh BK, Koh YX, et al. Validation of the MSKCC gastrointestinal stromal tumor nomogram and comparison with other prognostication systems: single-institution experience with 289 patients. Ann Surg Oncol. 2015;22(11):3597-3605.

18. ESMO/European Sarcoma Network Working Group. Gastrointestinal stromal tumours: ESMO Clinical Practice Guidelines for diagnosis, treatment and follow-up. Ann Oncol. 2014;25 Supp1 3:iii21-iii26. 
19. Demetri GD, Benjamin RS, Blanke CD, et al. NCCN Task Force report: management of patients with gastrointestinal stromal tumor (GIST)update of the NCCN Clinical Practice Guidelines. J Natl Compr Canc Netw. 2007;5(Suppl 2):S1-S31.

20. Fletcher CD, Berman JJ, Corless C, et al. Diagnosis of gastrointestinal stromal tumors: A consensus approach. Hum Pathol. 2002;33(5):459-465.

21. Gold JS, Gönen M, Gutiérrez A, et al. Development and validation of a prognostic nomogram for recurrence-free survival after complete surgical resection of localised primary gastrointestinal stromal tumour: a retrospective analysis. Lancet Oncol. 2009;10(11):1045-1052.

22. Rossi S, Miceli R, Messerini L, et al. Natural history of imatinib-naive GISTs: a retrospective analysis of 929 cases with long-term follow-up and development of a survival nomogram based on mitotic index and size as continuous variables. Am J Surg Pathol. 2011;35(11):1646-1656.

23. Bischof DA, Kim Y, Behman R, et al. A nomogram to predict diseasefree survival after surgical resection of GIST. $J$ Gastrointest Surg. 2014;18(12):2123-2129.

24. Schmieder M, Henne-Bruns D, Mayer B, et al. Comparison of different risk classification systems in 558 patients with gastrointestinal stromal tumors after R0-resection. Front Pharmacol. 2016;7:504.

25. Belfiori G, Sartelli M, Cardinali L, et al. Risk stratification systems for surgically treated localized primary gastrointestinal stromal tumors (GIST). Review of literature and comparison of the three prognostic criteria: MSKCC Nomogram, NIH-Fletcher and AFIP-Miettinen. Ann Ital Chir. 2015;86(3):219-227.

26. Doyle LA, Hornick JL. Gastrointestinal stromal tumours: from KIT to succinate dehydrogenase. Histopathology. 2014;64(1):53-67.

27. Miettinen M, Fetsch JF, Sobin LH, Lasota J. Gastrointestinal stromal tumors in patients with neurofibromatosis 1: a clinicopathologic and molecular genetic study of 45 cases. Am J Surg Pathol. 2006;30(1):90-96.

28. Zhou C, Duan X, Zhang X, Hu H, Wang D, Shen J. Predictive features of $\mathrm{CT}$ for risk stratifications in patients with primary gastrointestinal stromal tumour. Eur Radiol. 2016;26(9):3086-3093.

29. Miyake KK, Nakamoto Y, Mikami Y, et al. The predictive value of preoperative $18 \mathrm{~F}$-fluorodeoxyglucose $\mathrm{PET}$ for postoperative recurrence in patients with localized primary gastrointestinal stromal tumour. Eur Radiol. 2016;26(12):4664-4674.

30. Tokumoto N, Tanabe K, Misumi T, Fujikuni N, Suzuki T, Ohdan H. The usefulness of preoperative 18FDG positron-emission tomography and computed tomography for predicting the malignant potential of gastrointestinal stromal tumors. Dig Surg. 2014;31(2):79-86.

31. Bertucci F, Finetti P, Mamessier E, et al. PDL1 expression is an independent prognostic factor in localized GIST. Oncoimmunology. 2015;4(5):e1002729.

32. Suehara Y, Kondo T, Seki K, et al. Pfetin as a prognostic biomarker of gastrointestinal stromal tumors revealed by proteomics. Clin Cancer Res. 2008;14(6):1707-1717.

33. Orita H. Pfetin as a risk factor of recurrence in gastrointestinal stromal tumors. BioMed Res Int. 2014;651935.

34. Huang KK. SETD2 histone modifier loss in aggressive GI stromal tumours. Gut. 2016;65:1960-1972.

35. Wang CJ, Zhang ZZ, Xu J, et al. SLITRK3 expression correlation to gastrointestinal stromal tumor risk rating and prognosis. World $J$ Gastroenterol. 2015;21(27):8398-8407.

36. Gyvyte U. MiRNA profiling of gastrointestinal stromal tumors by nextgeneration sequencing. Oncotarget. 2017;8:37225-37238.

37. Li CF, Chuang IC, Liu TT, et al. Transcriptomic reappraisal identifies MGLL overexpression as an unfavorable prognosticator in primary gastrointestinal stromal tumors. Oncotarget. 2016;7(31):49986-49997.

38. Wozniak A, Rutkowski P, Schoffski P, et al. Tumor genotype is an independent prognostic factor in primary gastrointestinal stromal tumors of gastric origin: a European multicenter analysis based on ConticaGIST. Clin Cancer Res. 2014;20(23):6105-6116.

39. Rossi S, Gasparotto D, Miceli R, et al. KIT, PDGFRA, and BRAF mutational spectrum impacts on the natural history of imatinib-naive localized GIST: a population-based study. Am J Surg Pathol. 2015;39(7):922-930.
40. Alkhasawneh A, Reith JD, Toro TZ, et al. Interobserver variability of mitotic index and utility of $\mathrm{PHH} 3$ for risk stratification in gastrointestinal stromal tumors. Am J Clin Pathol. 2015;143(3):385-392.

41. Rizzo FM, Palmirotta R, Marzullo A, et al. Parallelism of DOG1 expression with recurrence risk in gastrointestinal stromal tumors bearing KIT or PDGFRA mutations. BMC Cancer. 2016;16:87.

42. Zhang Y, Gu ML, Zhou XX, Ma H, Yao HP, Ji F. Altered expression of ETV1 and its contribution to tumorigenic phenotypes in gastrointestinal stromal tumors. Oncol Rep. 2014;32(3):927-934.

43. Zhou Y, Hu W, Chen P, et al. Ki67 is a biological marker of malignant risk of gastrointestinal stromal tumors: a systematic review and metaanalysis. Medicine. 2017;96(34):34.

44. Stotz M, Liegl-Atzwanger B, Posch F, et al. Blood-based biomarkers are associated with disease recurrence and survival in gastrointestinal stroma tumor patients after surgical resection. PLoS One. 2016;11(7): $\mathrm{e} 0159448$

45. Xue A, Gao X, Fang Y, et al. Incorporation of NLR into NIH stratification system increases predictive accuracy for surgically resected gastrointestinal stromal tumors. Acta Biochim Biophys Sin. 2017;49(2):179-185.

46. Hsiao CY, Yang CY, Lai IR, Chen CN, Lin MT. Laparoscopic resection for large gastric gastrointestinal stromal tumor (GIST): intermediate follow-up results. Surg Endosc. 2015;29(4):868-873.

47. Lin J, Huang C, Zheng C, et al. Laparoscopic versus open gastric resection for larger than $5 \mathrm{~cm}$ primary gastric gastrointestinal stromal tumors (GIST): a size-matched comparison. Surg Endosc. 2014;28(9):2577-2583.

48. Piessen G, Lefèvre JH, Cabau M, et al. Laparoscopic versus open surgery for gastric gastrointestinal stromal tumors: what is the impact on postoperative outcome and oncologic results? Ann Surg. 2015;262(5):831-839.

49. Joensuu H, Eriksson M, Hall KS, et al. Risk factors for gastrointestinal stromal tumor recurrence in patients treated with adjuvant imatinib. Cancer. 2014;120(15):2325-2333.

50. Bischof DA, Dodson R, Jimenez MC, et al. Adherence to guidelines for adjuvant imatinib therapy for GIST: a multi-institutional analysis. J Gastrointest Surg. 2015;19(6):1022-1028.

51. Guérin A, Sasane M, Keir CH, et al. Physician underestimation of the risk of gastrointestinal stromal tumor recurrence after resection. JAMA Oncol. 2015;1(6):797-805.

52. Dematteo RP, Ballman KV, Antonescu CR, et al. Adjuvant imatinib mesylate after resection of localised, primary gastrointestinal stromal tumour: a randomised, double-blind, placebo-controlled trial. Lancet. 2009;373(9669):1097-1104.

53. Joensuu H, Eriksson M, Sundby Hall K, et al. One vs three years of adjuvant imatinib for operable gastrointestinal stromal tumor: a randomized trial. JAMA. 2012;307(12):1265-1272.

54. Trent JC, Subramanian MP. Managing GIST in the imatinib era: optimization of adjuvant therapy. Expert Rev Anticancer Ther. 2014;14(12):1445-1459.

55. Lin JX, Chen QF, Zheng CH, et al. Is 3-years duration of adjuvant imatinib mesylate treatment sufficient for patients with high-risk gastrointestinal stromal tumor? A study based on long-term follow-up. J Cancer Res Clin Oncol. 2017;143(4):727-734.

56. Casali PG, Le Cesne A, Poveda Velasco A, et al. Time to definitive failure to the first tyrosine kinase inhibitor in localized GI stromal tumors treated with imatinib as an adjuvant: a European Organisation for Research and Treatment of Cancer Soft Tissue and Bone Sarcoma Group Intergroup Randomized Trial in Collaboration with the Australasian Gastro-Intestinal Trials Group, UNICANCER, French Sarcoma Group, Italian Sarcoma Group, and Spanish Group for Research on Sarcomas. J Clin Oncol. 2015;33(36):4276-4283.

57. Joensuu H, Wardelmann E, Sihto H, et al. Effect of KIT and PDGFRA mutations on survival in patients with gastrointestinal stromal tumors treated with adjuvant imatinib: an exploratory analysis of a randomized clinical trial. JAMA Oncol. 2017;3(5):602-609.

58. Agaimy A, Wünsch PH, Hofstaedter F, et al. Minute gastric sclerosing stromal tumors (GIST tumorlets) are common in adults and frequently show c-KIT mutations. Am J Surg Pathol. 2007;31(1):113-120. 
59. Kawanowa K, Sakuma Y, Sakurai S, et al. High incidence of microscopic gastrointestinal stromal tumors in the stomach. Hum Pathol. 2006;37(12):1527-1535.

60. Rossi S, Gasparotto D, Toffolatti L, et al. Molecular and clinicopathologic characterization of gastrointestinal stromal tumors (GISTs) of small size. Am J Surg Pathol. 2010;34(10):1480-1491.

61. Coe TM, Fero KE, Fanta PT, et al. Population-based epidemiology and mortality of small malignant gastrointestinal stromal tumors in the USA. J Gastrointest Surg. 2016;20(6):1132-1140.

62. Nishida T, Hirota S, Yanagisawa A, et al. Clinical practice guidelines for gastrointestinal stromal tumor (GIST) in Japan: English version. Int J Clin Oncol. 2008;13(5):416-430.

63. Nishida T, Goto O, Raut CP, Yahagi N. Diagnostic and treatment strategy for small gastrointestinal stromal tumors. Cancer. 2016;122(20):3110-3118.
64. Palazzo L, Landi B, Cellier C, Cuillerier E, Roseau G, Barbier JP. Endosonographic features predictive of benign and malignant gastrointestinal stromal cell tumours. Gut. 2000;46(1):88-92.

65. Chak A, Canto MI, Rösch T, et al. Endosonographic differentiation of benign and malignant stromal cell tumors. Gastrointest Endosc. 1997;45(6):468-473.

66. Demetri GD, von Mehren M, Antonescu CR, et al. NCCN Task Force report: update on the management of patients with gastrointestinal stromal tumors. J Natl Compr Canc Netw. 2010;8(Suppl 2): S1-S10.

67. Agaimy A. Gastrointestinal stromal tumor (GIST) from risk stratification systems to the new TNM proposal: more questions than answers? A review emphasizing the need for a standardized GIST reporting. Int J Clin Exp Pathol. 2010;3(5):461-471.
Gastrointestinal Cancer: Targets and Therapy

\section{Publish your work in this journal}

Gastrointestinal Cancer: Targets and Therapy is an international, peer-reviewed, open access journal focusing on gastro-intestinal cancer research, identification of therapeutic targets and the optimal use of preventative and integrated treatment interventions to achieve improved outcomes, enhanced survival and quality of life for the
Dovepress

cancer patient. The manuscript management system is completely online and includes a very quick and fair peer-review system. Visit http://www.dovepress.com/testimonials.php to read real quotes from published authors.

Submit your manuscript here: https://www.dovepress.com/gastro-intestinal-cancer-targets-and-therapy-journal 